Professor Roemer, of Marburg, has been called to Greifswald to conduct the hygienic institute as the successor of Professor Loeffler.

\section{DISCOSSION AND CORRESPONDENCE}

A NEW TYPE OF BACTERIAL DISEASE

By this title I mean a disease in which the bacterial growth first develops conspicuously as a thick layer on the surface of the plant, and only later penetrates into its interior.

Ráthay's disease of orchard grass (Dactylis glomerata) described by him in 1899 may be taken as the type of this kind of disease. In 1913 I had opportunity to verify Ráthay's statements $^{1}$ on material sent to me from Denmark by Professor $K_{\phi}$ lpin Ravn, and to make pure cultures and further studies of the organism which in honor of Ráthay, may be known as Aplanobacter ráthayi $\mathrm{n}$. sp., with the characters assigned to it by Ráthay, and in addition the following:

Nitrates are not reduced; gelatin is finally liquefied, but liquefaction is visible only after some weeks and progresses very slowly; it does not grow in Cohn's solution; growth starts off slowly in milk, but is prolonged with formation of a copious chrome yellow precipitate and a wide bright yellow rim; litmus milk is first slowly blued, but becomes purplish after some weeks; it grows so slowly on agar that poured plates which appear to be sterile may eventually give small yellow colonies. Nearly all of Ráthay's statements have been found to be correct. This note is here published because of delay in the issue of a longer account.

ERwin F. Smith

THE MANUS OF TRACHODONT DINOSAURS

IN a recent article in The Ottawa Naturalist, Mr. Lawrence M. Lambe has described "The Manus in a Specimen of Trachodon from the Edmonton of Alberta," illustrated by three figures. According to Mr. Lambe's interpretation of the Ottawa skeleton the phalangeal formula is as follows:

1 Sitz. Ber. Wiener Akad., 1 Abt., Bd. CVIII., p. 597.

1 Vol. XXVII., pp. 21-25, 1913.
Digit II. with three phalanges, the third bearing a hoof.

Digit III. with three phalanges, the third bearing a hoof.

Digit IV. with two phalanges, the second bearing a hoof.

Digit V. with two phalanges, the second bearing a hoof.

Whereas in a specimen that I have described the formula is

Digit II. with three phalanges, the third bearing a hoof.

Digit III. with three phalanges, the third bearing a hoof.

Digit IV. with three phalanges, the third a vestigial bone without hoof.

Digit V. with three phalanges, the third a vestigial bone without hoof.

The writer published a description of the manus of Trachodon annectens, ${ }^{2}$ based on the first reported specimen in which all of the phalanges are present. In this specimen the full number of phalanges are not only present but each digit is articulated either in the right or the left hand and all are encased in a thin layer of matrix in which the skin impression is preserved.

In this uncrushed specimen the long slender metacarpals of digits II., III., and IV. are closely appressed as represented in the figure accompanying the above article, a position verified by structure and by position in three other uncrushed specimens in the American $\mathrm{Mu}$ seum, one in the National Museum, and a sixth in the collection of the Calgary Natural History Society.

In no specimen of the genus Trachodon known to me have more than two hoof bones been found in the manus-those of digits II. and III. The terminal phalanges of digits IV. and $\nabla$. are, when uncrushed, rounded bony nodules, very much reduced and were not covered by a hoof or nail.

If Mr. Lambe's interpretation is correct we have a remarkable specific variation in this genus in which a later species, described by me, has developed an additional phalanx on each

2 Bull. Am. Mus. Nat. Hist., Vol. XXXI., Art. X., pp. 105-107, 1912. 